\title{
Biopsia pulmonar por minitoracotomía. ¿Es necesario el drenaje pleural?
}

\author{
Servio Tulio Torres-Rodríguez,凶 Danilo Herrera-Cruz, Luis López-Yepes, Elka Lainfiesta-Moncada
}

Hospital San Vicente, Guatemala, Guatemala.

\begin{abstract}
RESUMEN. La utilidad de la biopsia pulmonar abierta en la obtención de material tisular para diagnóstico histológico es innegable, aun cuando los avances tecnológicos nos encauzan hacia los métodos de mínima invasión. El objetivo del estudio es mostrar la eficiencia de la biopsia pulmonar abierta y debatir la necesidad del drenaje pleural cuando la pleura ha sido transgredida. Material y métodos: Estudio prospectivo longitudinal realizado del 01 de enero del 2017 al 31 de diciembre del 2018, en 41 pacientes intervenidos de biopsia pulmonar abierta por minitoracotomía. Resultados: El estudio incluyó 41 pacientes sometidos a biopsia pulmonar abierta. La evolución fue satisfactoria en el 97.5\% de los casos y únicamente un paciente requirió la colocación de drenaje posoperatorio inmediato por neumotórax mayor del 10\%. En 39 (95\%) pacientes se obtuvo diagnóstico histológico independiente del sitio de la toma de biopsia y en dos (5\%) se reportó la muestra sin anormalidades. Conclusiones: La elección del sitio de toma de biopsia del parénquima pulmonar y la localización anatómica de la lesión no es impedimento para el abordaje por minitoracotomía y permite obtener tejido pulmonar adecuado para diagnóstico histológico en la mayoría de los casos. La indicación del drenaje pleural puede ser selectiva de acuerdo con criterios transoperatorios y de la experiencia del equipo quirúrgico.
\end{abstract}

Palabras clave: Biopsia pulmonar, minitoracotomía, drenaje pleural.

\section{Lung biopsy by minithoracotomy. Is the pleural drainage necessary?}

ABSTRACT. The usefulness of open lung biopsy in obtaining tissue material for histological diagnosis is undeniable even when technological advances direct us towards minimally invasive methods. The aim of the study is to show the efficiency of open lung biopsy and to discuss the need for pleural drainage when the pleura has been transgressed. Material and methods: Prospective longitudinal study carried out from January 1, 2017 to December 31, 2018, in 41 patients undergoing open lung biopsy by mini-thoracotomy. Results: The study included 41 patients with open lung biopsies. The evolution was satisfactory in $97.5 \%$ of the cases and only one patient required the placement of immediate postoperative drainage due to pneumothorax greater than 10\%. In 39 (95\%) patients, histological diagnosis was obtained independent of the site of the biopsy and two (5\%) the sample was reported without abnormalities. Conclusions: The choice of the lung parenchyma biopsy site and the anatomic location of the lesion is not an impediment to the mini-thoracotomy approach and allows obtaining adequate lung tissue for histological diagnosis in the majority of cases. The indication of pleural drainage can be selective according to transoperative criteria and the experience of the surgical team.

Key words: Lung biopsy, minithoracotomy, pleural drainage.

\section{INTRODUCCIÓN}

El avance tecnológico en la medicina con el advenimiento y las indicaciones cada vez más amplias de la videotoracoscopia ofreciendo grandes ventajas, tanto para el paciente que la recibe como para el médico que la utiliza, contrasta con lo que por tradición se ha hecho con la biopsia pulmonar abierta. Este procedimiento realizado

\section{$\triangle$ Autor para correspondencia:}

Dr. Servio Tulio Torres-Rodríguez, Hospital San Vicente,

Guatemala, Guatemala.

Correo electrónico: stuliotr@gmail.com

Trabajo recibido: 11-XII-2018; aceptado: 19-XII-2018 por una minitoracotomía, que no requiere del equipo de video ni de los accesorios de engrapado, continúa siendo una excelente opción para aquellos centros donde la tecnología de la toracoscopia no ha sido obtenida, logrando la obtención de tejido pulmonar capaz de suministrarle al patólogo la cantidad de material adecuado para llegar al diagnóstico histopatológico. El objetivo del estudio es mostrar la eficiencia de la biopsia pulmonar abierta por minitoracotomía y debatir la necesidad del drenaje pleural una vez que la pleura ha sido abierta.

\section{MATERIAL Y MÉTODOS}

Estudio prospectivo longitudinal realizado durante dos años, del 01 de enero del 2017 al 31 de diciembre del 


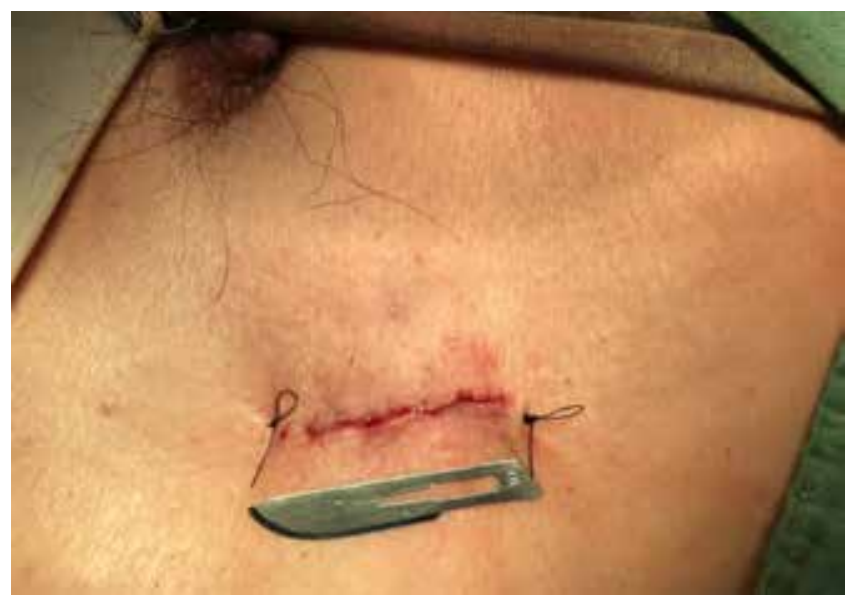

Figura 1: Tamaño de la incisión, tomando como referencia hoja de bisturí número 10.

2018, en 41 pacientes adultos sometidos a biopsia pulmonar por minitoracotomía sin drenaje pleural. Se incluyeron parámetros de edad, sexo, hemitórax afectado, sitio de toma de biopsia, evolución clínica, evolución radiológica, infección de herida quirúrgica, resultados de histopatología y mortalidad a 30 días.

\section{Criterios de inclusión}

Pacientes sin diagnóstico clínico o etiología incierta de infiltrados, consolidaciones o masas pulmonares a pesar de múltiples pruebas funcionales y de laboratorio, o aquéllos con diagnósticos establecidos, pero con necesidad de ajuste del tratamiento. Pacientes que presentaran en el transoperatorio las siguientes características: a) pulmones de consistencia blanda con expansibilidad adecuada, b) sin adherencias pleuropulmonares que requirieran disección extensa, ni engrosamiento pleural, c) fugas aéreas observadas a maniobras de Valsalva mientras se vertía agua en la línea de sutura y d) que no presentaran derrame pleural.

\section{Criterios de exclusión}

La manifestación de uno o más de estos criterios impone la colocación de drenaje pleural.

\section{Procedimiento de la biopsia pulmonar por minitoracotomía}

Todos los procedimientos se realizaron en la sala de operaciones por el mismo equipo quirúrgico conformado por un cirujano torácico y un cirujano general con entrenamiento en tórax, bajo anestesia general con intubación bronquial selectiva. La elección del sitio de toma de biopsia se determinó por la presencia de mayor patología pulmonar observada en la tomografía de tórax indicada a todos los pacientes. A través de una incisión de 3 a 5 $\mathrm{cm}$ en el hemitórax elegido, se tomó biopsia pulmonar de $3 \times 4 \mathrm{~cm}$ de diámetro, orientándola al lóbulo pulmonar mayormente afectado, sin importar su localización, fuera apical, anterior, posterior o basal (figura 1).

En los casos de sospecha de fibrosis pulmonar, la muestra se obtuvo con el pulmón expandido aplicando dos pinzas de Karmal y sección entre ellas (figuras 2A-2C). La técnica de cierre de la sección del pulmón consistió en sutura de material no absorbible de ácido poliglicólico 3(0) en surjete lineal continuo por debajo de la pinza y una segunda línea de sutura con Vicryl ${ }^{\circledR}$.
Figura 2:

A) Imagen tomográfica.

B) Toma de biopsia entre pinzas de Karmal.

C) Biopsia pulmonar.
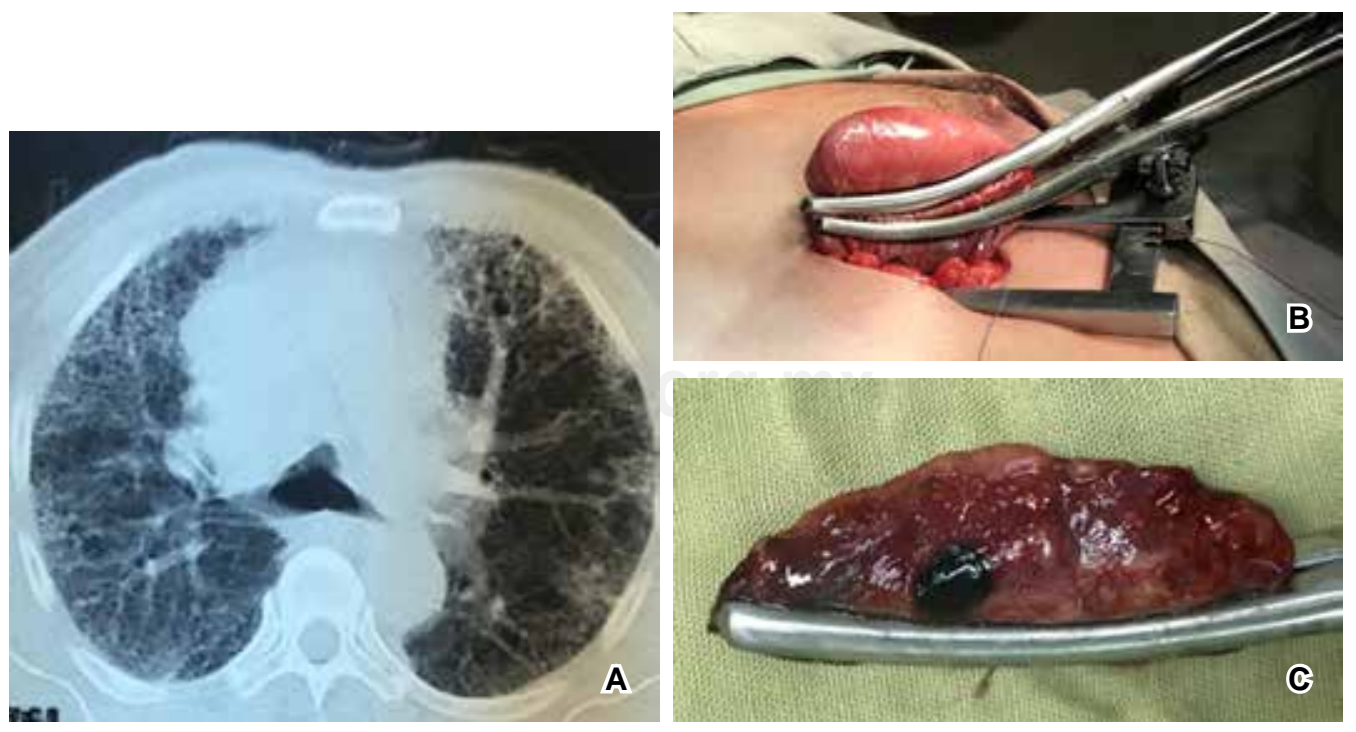
Figura 3:

A) Surjete continuo lineal.

B) Surjete continuo pa-

sado.
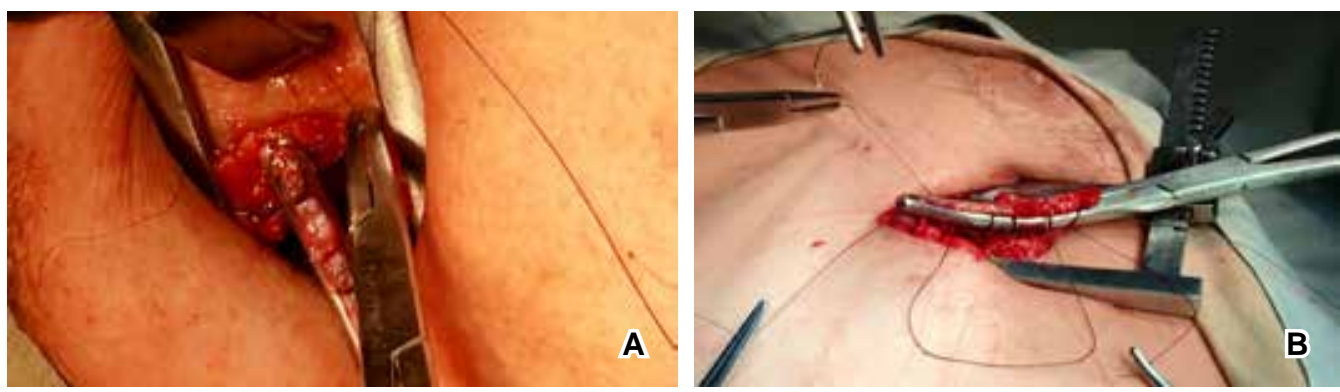

3(0) en surjete continuo pasado incluyendo la pinza (figuras 3A y 3B). Al final se tensan los extremos y se anudan entre sí. Se hace prueba de cierre hermético, vertiendo agua sobre la sutura mientras el anestesiólogo da un Valsalva.

La maniobra para evacuar el neumotórax inicial se efectúa a través de una sonda de alimentación \#8, introducida por incisión contralateral, aplicando succión mientras se cierran los planos de apertura del tórax. Antes de retirar la sonda, se solicitan maniobras de Valsalva y se retira completamente manteniendo la expansión pulmonar (figura 4).

Una vez obtenido el espécimen, se hicieron extendidos para Gram y Ziehl-Neelsen, luego se fijó el tejido en formalina al $10 \%$ enviándola para estudio de histopatología. La realización de pruebas de inmunohistoquímica estuvo sujeta al diagnóstico primario reportado y a las condiciones económicas del paciente para el pago del estudio (figura 5).

\section{Seguimiento}

A todos los pacientes se les controló clínicamente por medio de la visita médica, observando presencia o ausencia de enfisema subcutáneo, disnea, cianosis y alteración del estado general. A todos los pacientes se

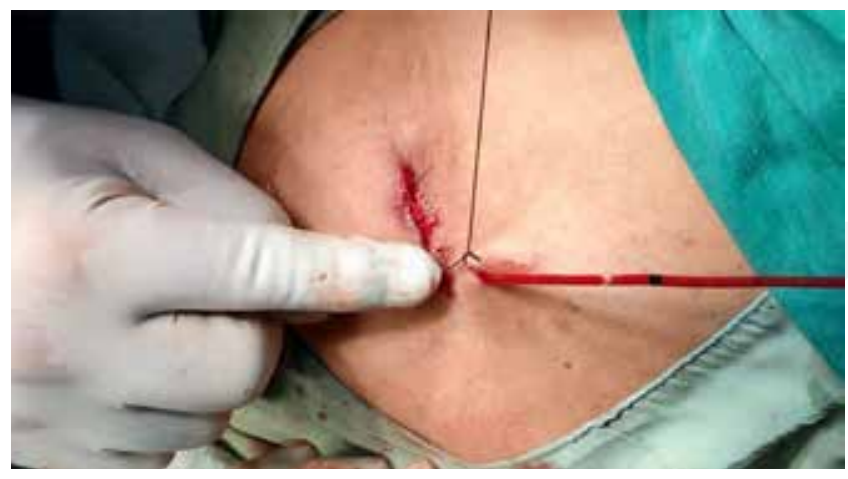

Figura 4: Succión del neumotórax inicial con sonda de alimentación \#8.

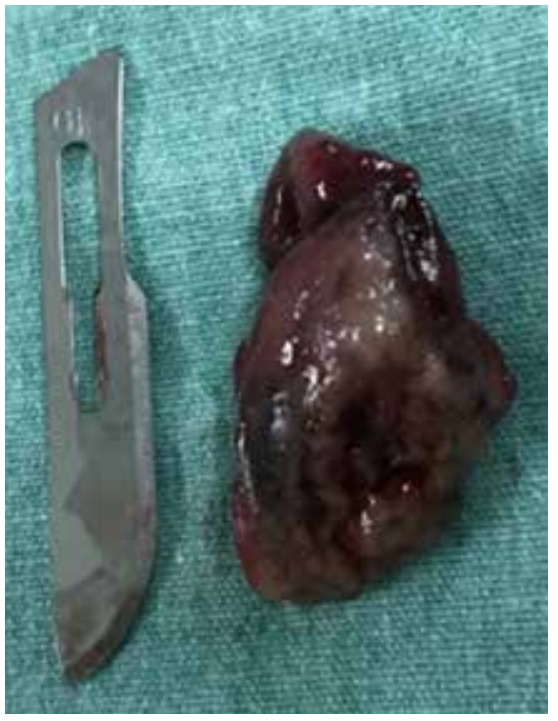

Figura 5:

Tamaño de la biopsia comparada con una hoja de bisturí \#10.

les solicitó radiografía de tórax a las cuatro horas y a las 24 horas posoperatorias en busca de la presencia de neumo o hemotórax en los estudios radiológicos.

\section{RESULTADOS}

Del 1 de enero del 2017 al 31 de diciembre del 2018 se realizaron 55 biopsias pulmonares por minitoracotomía, de los cuales a 41 (75\%) no se les dejó drenaje pleural. Veintidós pacientes (54\%) correspondieron al sexo femenino y la edad promedio para ambos sexos fue de 53 años (DE 50.91).

En todos los pacientes se obtuvo material tisular adecuado que llevó en 39 (95\%) a un diagnóstico histológico independiente del sitio de la toma de biopsia y en dos (5\%) se reportó la muestra sin anormalidades. La língula, por las ventajas que ofrece en accesibilidad, fue la más utilizada de todos los procedimientos en 16 pacientes (39\%), seguidos por el lóbulo superior en nueve casos (15\%) y el lóbulo medio en seis (figura 6).

Se presentaron complicaciones en 6/41 (14.63\%) pacientes, siendo la más grave la de un paciente que presentó paro cardíaco reversible en transoperatorio. 


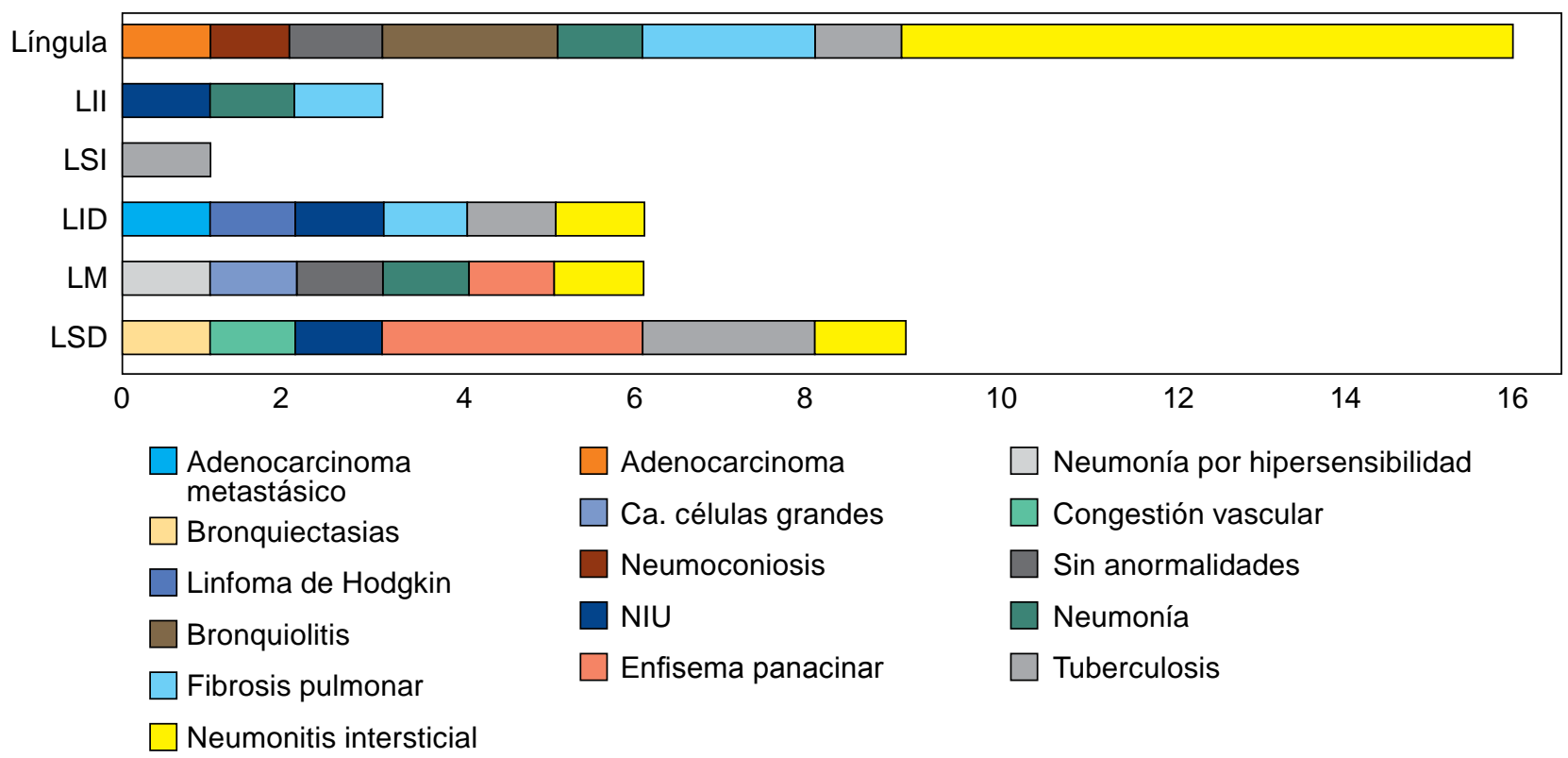

Figura 6: Diagnósticos obtenidos en relación al sitio anatómico de toma de biopsia.

Abreviaturas: LII: lóbulo inferior izquierdo; LSI: lóbulo superior izquierdo; LID: lóbulo inferior derecho; LM: lóbulo medio; LSD: Ióbulo superior derecho.

Otro paciente desarrolló edema pulmonar agudo en el posoperatorio inmediato. En tres pacientes (7.31\%) se presentó neumotórax menor del $5 \%$ que no requirió ninguna intervención y uno, (2.43\%) neumotórax mayor del $10 \%$ que demandó la colocación de drenaje pleural a través de un tubo intercostal. No reportamos infección de herida quirúrgica ni mortalidad a 30 días posoperatorios.

La patología benigna se diagnosticó en el $90 \%$ de los casos, siendo el intersticio el más afectado, manifestado ya sea como neumonitis, fibrosis pulmonar o neumonitis intersticial usual (NIU) en 17 pacientes (41.36\%), seguida de enfermedad infecciosa en 11 (29.26\%), de los cuales la tuberculosis pulmonar se encontró en cinco pacientes. El diagnóstico de neoplasia primaria o metastásica se obtuvo en cuatro (9.75\%) (tabla 1).

\section{DISCUSIÓN}

El objetivo de la biopsia pulmonar es la obtención de tejido pulmonar en cantidad y calidad adecuada para llegar a un diagnóstico preciso; y las opciones para su obtención van desde las punciones de mínima invasión transtorácicas o broncoscópicas hasta las más complejas como la biopsia abierta, considerada como el estándar de oro y la videotoracoscopia, que, en la actualidad, día con día la va desplazando.

Todas las biopsias pulmonares fueron realizadas a través de una minitoracotomía de 3 a $5 \mathrm{~cm}$ de longitud
Tabla 1: Diagnósticos obtenidos en biopsia pulmonar por minitoracotomía.

\begin{tabular}{|l|c|}
\hline Neumonitis intersticial & 10 \\
\hline Tuberculosis pulmonar & 5 \\
\hline Fibrosis pulmonar & 4 \\
\hline Enfisema panacinar & 4 \\
\hline Neumonitis intersticial usual & 3 \\
\hline Neumonía & 3 \\
\hline Sin anormalidades & 2 \\
\hline Bronquiolitis & 2 \\
\hline Neumonía por hipersensibilidad & 1 \\
\hline Neumoconiosis & 1 \\
\hline Linfoma de Hodgkin & 1 \\
\hline Congestión vascular & 1 \\
\hline Carcinoma de células grandes & 1 \\
\hline Bronquiectasias & 1 \\
\hline Adenocarcinoma & 1 \\
\hline Adenocarcinoma metastásico & 1 \\
\hline Total & 41 \\
\hline
\end{tabular}

en el hemitórax donde radiológicamente se observara mayor patología por TAC, evitando regiones intensamente afectadas por la fibrosis, particularmente en panal de abejas, pues estos sitios revelan cambios terminales de fibrosis con poco valor diagnóstico. ${ }^{1}$ En todos ellos se obtuvo tejido pulmonar en cantidad 
adecuada para el diagnóstico independiente de la localización dentro del tórax, lo que permitió el inicio de una terapéutica o una readecuación de cambios en el manejo de los pacientes, tal como ha sido reportado. ${ }^{2-7}$

Ninguno de nuestros pacientes presentó criterios para ventilación mecánica ni intubación endotraqueal previa o posterior a la finalización del procedimiento. Sin embargo, la contribución de la biopsia pulmonar abierta en la mejoría de la sobrevivencia de pacientes con dificultad respiratoria aguda ha sido ampliamente reportada ${ }^{8-10}$ y para obtener un beneficio máximo debe ser reservada en pacientes con pronóstico razonable y no en pacientes moribundos. ${ }^{11}$ En la enfermedad pulmonar intersticial su indicación es cada vez mayor, con una mortalidad a 30 y 90 días del 1.7 y $2.4 \%$ en grandes series, ${ }^{12}$ y se incrementa cuando se asocia a daño alveolar difuso. ${ }^{13}$ Nuestra mortalidad a 30 y 90 días fue del $0 \%$ y el diagnóstico mayormente obtenido fue de patología intersticial, seguida de tuberculosis pulmonar, que, si bien no se aisló el bacilo de Koch, los hallazgos histopatológicos reportaron presencia de células gigantes de Langerhans, necrosis caseosa, histiocitos y en algunos casos granulomas. A pesar que estos pacientes tenían reacción en cadena de la polimerasa (PCR, por sus siglas en inglés) y baciloscopia negativa, la situación geográfica de zona endémica nos indujo a iniciar tratamiento antifímico.

La biopsia por minitoracotomía es un procedimiento con baja tasa de complicaciones relacionadas al procedimiento en sí. Éstas se presentaron en 6/41 pacientes (14.63\%), siendo la más grave la de un paciente que presentó paro cardíaco reversible en transoperatorio; fue el único en quien se utilizó sutura mecánica en la sección del parénquima pulmonar con engrapadora endoGIA por la urgencia del caso. Otro paciente desarrolló edema pulmonar agudo en el posoperatorio inmediato. Ambos pacientes se recuperaron completamente y no presentaron secuelas en su evolución. Tres pacientes (7.31\%) presentaron neumotórax menor del $5 \%$ que no requirió ninguna intervención y uno, (2.43\%) neumotórax mayor del 10\% que requirió la colocación de drenaje pleural. En series más grandes, las complicaciones no letales reportadas se enmarcan sobre todo en la fuga aérea prolongada hasta en un $5 \%{ }^{14}$ y cuando se compara con las realizadas por videotoracoscopia asistida (VATS, por sus siglas en inglés), éstas van del $9.6 \%$ en toracoscopia al $18 \%$ en biopsia abierta..$^{15}$

Tradicionalmente, una vez que la pleura se abre, su cierre se acompaña con la colocación de drenaje a través de una sonda intratorácica. Existen pocos reportes de realizar un procedimiento abierto sin colocación de drenaje torácico en resecciones lingulares. ${ }^{16}$ Común- mente las publicaciones de resección en cuña e incluso lobectomías sin tubo torácico sin complicaciones severas se refieren a los realizados por videotoracoscopia, con las ventajas de menos dolor, pequeñas incisiones y menor trauma, ${ }^{17,18}$ y su uso innecesario incrementa la estadía hospitalaria sin reducción de la incidencia de neumotórax. ${ }^{19}$ Del total de biopsias pulmonares por minitoracotomía, decidimos no dejar drenaje pleural en aquellos pacientes que cumplieran los siguientes criterios: a) pulmones de consistencia blanda, b) expansibilidad adecuada, c) adherencias pleuropulmonares laxas o inexistentes, d) sin engrosamiento pleural, e) no fugas aéreas observadas a maniobras de Valsalva mientras se vierte agua en la línea de sutura y f) ausencia de derrame pleural.

En todos los pacientes se realizó sección y sutura manual del parénquima pulmonar con material absorbible de ácido poligláctico 3(0), con aguja atraumática. Se comprobó impermeabilidad del pulmón con prueba de agua. La evolución fue satisfactoria en el $97.5 \%$ de los casos y únicamente un paciente requirió la colocación de drenaje posoperatorio inmediato por neumotórax mayor del $10 \%$. Nuestros resultados proponen que este procedimiento puede ser recomendable por su seguridad y bajo costo al no incurrir en gasto extra por equipo de video, accesorios de engrapado ni del kit de drenaje torácico. El carecer de un grupo control aleatorizado es la principal limitación del trabajo.

\section{CONCLUSIÓN}

La biopsia pulmonar abierta por minitoracotomía es un procedimiento que permite obtener tejido pulmonar adecuado para diagnóstico histológico independientemente de su localización dentro del tórax y los resultados obtenidos sugieren que el criterio de colocar drenaje pleural puede ser selectivo si se cumple con los criterios propuestos y basados en la experiencia de cada centro y de su equipo.

\section{Conflicto de intereses}

Los autores declaran no tener conflicto de intereses.

\section{REFERENCIAS}

1. Raj R, Raparia K, Lynch DA, Brown KK. Surgical lung biopsy for interstitial lung diseases. Chest 2017;151(5):1131-1140. doi: 10.1016/j.chest.2016.06.019.

2. Blackhall V, Asif M, Renieri A, et al. The role of surgical lung biopsy in the management of interstitial lung disease: Experience from a single institution in the UK. Interact Cardiovasc Thorac Surg 2013;17(2):253-257. doi: 10.1093/icvts/ivt217. 
3. Torres-Rodríguez T, Aburto A, Morales GJ, Téllez JL, Melchum L, Alejandre JVJ. La biopsia pulmonar a cielo abierto. ¿Modifica la terpéutica final? Rev Inst Nal Enf Resp Méx 1991;4:59.

4. Donaldson LH, Gill AJ, Hibbert M. Utility of surgical lung biopsy in critically ill patients with diffuse pulmonary infiltrates: a retrospective review. Intern Med J 2016;46(11):1306-1310. doi: 10.1111/imj.13222.

5. Marjański T, Halman J, Taniewska S, Burzyńska N, Piekarska A, Sawicka W. Open lung biopsy performed in idiopathic pulmonary fibrosis is a safe procedure. Kardiochir Torakochirurgia Pol 2017;14(4):236-240. doi: 10.5114/kitp.2017.72227.

6. Blewett CJ, Bennett WF, Miller JD, Urschel JD. Open Lung biopsy as an outpatient procedure. Ann Thorac Surg 2001;71(4):1113-1115. doi: 10.1016/S00034975(00)02657-6.

7. Libby LJ, Gelbman BD, Altorki NK, Christos PJ, Libby DM. Surgical lung biopsy in adult respiratory distress syndrome: A meta-analysis. Ann Thorac Surg 2014;98(4):1254-1260. doi: 10.1016/j. athoracsur.2014.05.029.

8. Papazian L, Doddoli C, Chetaille B, et al. A contributive result of open-lung biopsy improves survival in acute respiratory distress syndrome patients. Crit Care Med 2007;35(3):755-762. doi: 10.1097/01. CCM.0000257325.88144.30.

9. Kramer MR, Berkman N, Mintz B, Godfrey S, Saute M, Amir $\mathrm{G}$. The role of open lung biopsy in the management and outcome of patients with diffuse lung disease. Ann Thorac Surg 1998;65(1):198-202.

10. Arabi $Y$, Ahmed $R$, Ahmed $Q$, Rahman MU, Yamani $\mathrm{N}$. Risks and benefits of open-lung biopsy in the mechanically ventilated critically ill population: a cohort study and literature review. Med Sci Monit 2007;13(8):CR365-CR371.

11. Almotairi A, Biswas S, Shahin J. The role of open lung biopsy in critically III patients with hypoxic respiratory failure: a retrospective cohort study. Can Respir J 2016;2016:8715024. doi: 10.1155/2016/8715024.
12. Hutchinson JP, McKeever TM, Fogarty AW, Navaratnam V, Hubbard RB. Surgical lung biopsy for the diagnosis of interstitial lung disease in England: 1997-2008. Eur Respir J 2016;48(5):1453-1461. doi: 10.1183/13993003.003782016.

13. Kao KC, Chang $\mathrm{CH}$, Hung $\mathrm{CY}$, Chiu LC, Huang $\mathrm{CC}, \mathrm{Hu} \mathrm{HC}$. Survival predictor in patients with acute respiratory distress syndrome and diffuse alveolar damage undergoing open lung biopsy. PLoS One 2017;12(7):e0180018. doi: 10.1371/journal. pone.0180018.

14. Rotolo N, Imperatori A, Dominioni L, et al. Efficacy and safety of surgical lung biopsy for interstitial disease. Experience of 161 consecutive patients from a single institution in Italy. Sarcoidosis Vasc Diffuse Lung Dis 2015;32(3):251-258.

15. Nguyen W, Meyer KC. Surgical lung biopsy for the diagnosis of interstitial lung disease : a review of the literature and recommendations for optimizing safety and efficacy. Sarcoidosis Vasc Diffuse Lung Dis 2013;30(1):3-16.

16. Koç T, Routledge T, Chambers A, Scarci M. Do patients undergoing lung biopsy need a postoperative chest drain at all? Interact Cardiovasc Thorac Surg 2010;10(6):1022-1025. doi: 10.1510/icvts.2010.232892.

17. Watanabe A, Watanabe T, Ohsawa H, et al. Avoiding chest tube placement after video-assisted thoracoscopic wedge resection of the lung. Eur J Cardiothorac Surg 2004;25(5):872-876. doi: 10.1016/j.ejcts.2004.01.041.

18. Lu TY, Chen JX, Chen PR, et al. Evaluation of the necessity for chest drain placement following thoracoscopic wedge resection. Surg Today 2017;47(5):606-610. doi: 10.1007/s00595-016-1414-5.

19. Satherley LK, Luckraz H, Rammohan KS, Phillips M, Kulatilake NE, O'Keefe PA. Routine placement of an intercostal chest drain during video-assisted thoracoscopic surgical lung biopsy unnecessarily prolongs in-hospital length of stay in selected patients. Eur J Cardiothorac Surg 2009;36(4):737-740. doi: 10.1016/j.ejcts.2009.04.067. 